\title{
Relationship Between Rockwell C Hardness and Inelastic Material
}

\section{Constants}

\author{
Akihiko Hirano $^{1}$, Masao Sakane ${ }^{2}$ and Naomi Hamada ${ }^{3}$ \\ ${ }^{1}$ Department of Automotive Engineering Faculty of Junior College of Automotive Industry, \\ Osaka Sangyo University, Junior College \\ 3-1-1, Nakagaito, Daito-shi, Osaka, 574-8530, Japan \\ ${ }^{2}$ Department of Mechanical Engineering, Faculty of Science and Engineering, \\ Ritsumeikan University \\ 1-1-1, Nojihigashi, Kusatsu-shi, Shiga, 525-8577, Japan \\ ${ }^{3}$ Department of Mechanical Engineering, Hiroshima Kokusai Gakuin University \\ 16-21, Nakano, Aki-ku, Hiroshima-shi, Hiroshima, 739-0321, Japan
}

\begin{abstract}
This paper describes the relationship between Rockwell $\mathrm{C}$ hardness and elastic-plastic material constants by using finite element analyses. Finite element Rockwell $\mathrm{C}$ hardness analyses were carried out to examine the effects of friction coefficient and elastic-plastic material constants on the hardness. The friction coefficient and Young's modulus have no influence on the hardness but the inelastic materials constants, yield stress and strain hardening coefficient and exponent, have a significant influence on the hardness. A new equation for predicting the hardness was proposed as a function of yield stress and strain hardening coefficient and exponent. The equation evaluated the hardness within a $\pm 5 \%$ difference for all the finite element and experimental results.
\end{abstract}

\section{KEYWORDS}

FEM analisis, Rockwell C hardness, Inelastic constitutive relationship, Strain hardening coefficient, Strain hardening exponent

\section{INTRODUCTION}

Hardness testing is one of the most frequent to material testings and is understood as a testing to measure the resistance to compressive permanent deformation. However, the physical meaning of hardness has been an open question. Especially, inelastic material contains are supposed to have some connection but a quantitative relationship has not been well defined. Experimental studies tried to obtain the quantitative relationship between the materials constants and hardness using many different materials that have different tensile properties, but they did not succeed because every material constant changes when using a different material [1]. To develop a quantitative relationship, an analytical method of changing only a specific material constant is needed keeping the other constants unchanged. Recent development finite element (FE) contact analysis enables this . The authors applied this approach to Brinell hardness and proposed a quantitative relationship [3]. Rockwell $\mathrm{C}$ hardness (HRC), however, is a representative hardness testing and a quantitative equation has been also needed. This paper studies the relationship between Rockwell $\mathrm{C}$ 
hardness and the elastic-plastic material constants using finite element analyses. Finite element contact analyses were made by varying yield stress and strain hardening coefficient and exponent. Based on the quantitative relationship between HRC and those material constants, an equation predicting the hardness from the material constants well be proposed. The accuracy of the equation well be discussed by comparing the hardness between the prediction, FE analysis and experiments.

\section{RESULTS AND DISCUTION}

\section{Finite Element Model}

Figure 1 shows the FE meshes used for the analysis. An axi-symmetric 2D isoparametric 4-nodes element was used to obtain the relationship between HRC and the inelastic material constants, Figure 1 (a).

The same mesh was used for analyzing the critical thickness for the hardness testing. An isoparametric 3D 8-nodes element was used for the analysis of the critical distance from specimen edge, Figure 2 (b). A quarter part of a whole model was meshed in 3D analysis from the symmetry of the model. The circular

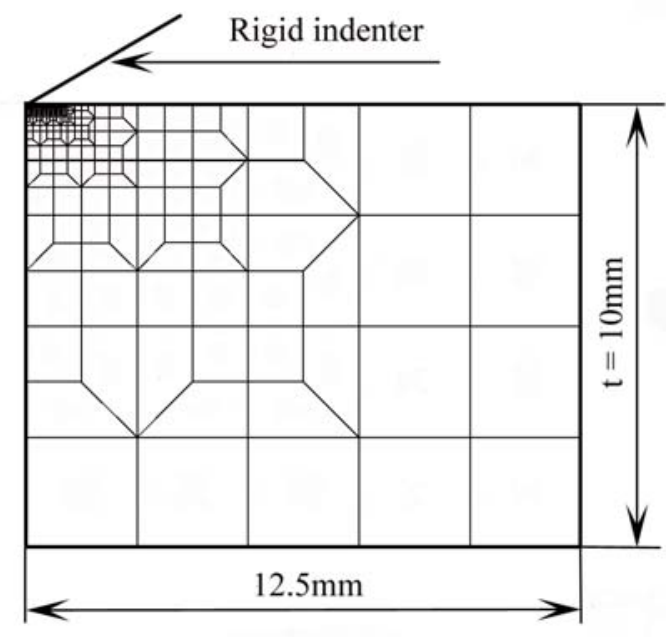

(a) $2 \mathrm{D}$ mesh

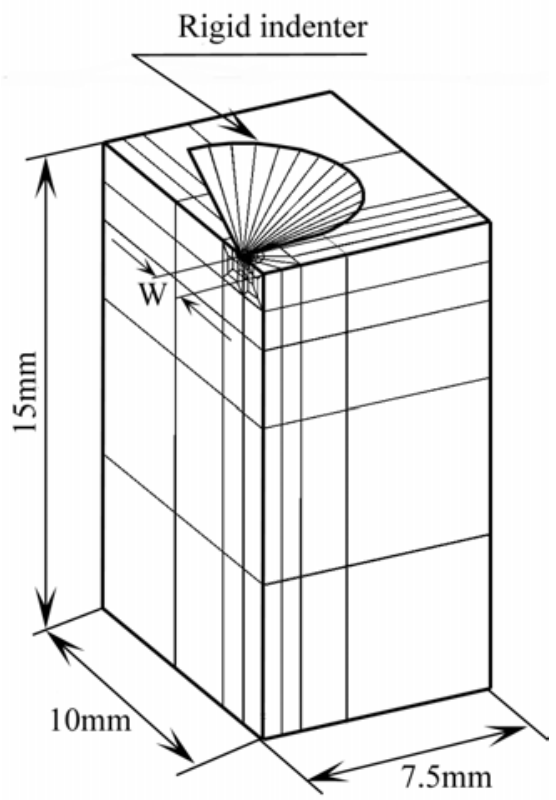

(b) $3 \mathrm{D}$ mesh

Fig.1 FEM meshes used for analyses.

conical indenter with a flank angle of 120 degrees and $0.2 \mathrm{~mm}$ tip radius was modeled to a rigid body in both meshes based on the previous result that the elastic deformation of the indenter has no effect on Brinell hardness [3]. The numbers of nodes and elements are 773 and 727 in the 2D mesh, and those of nodes and elements are 3785 and 2136 in the 3D mesh, respectively. Thickness, $t$, in Figure 1 (a) was changed from $0.156 \mathrm{~mm}$ to $5.00 \mathrm{~mm}$ and the edge distance, w, in Figure 1 (b) from $0.45 \mathrm{~mm}$ to $5.00 \mathrm{~mm}$.

In FE analysis, load of $98 \mathrm{~N}$ was initially applied, increased to the maximum value of $1471 \mathrm{~N}$ and decreased to $98 \mathrm{~N}$ to simulate $\mathrm{HRC}$ testing. The relative indenter displacement $\left(\delta^{*}\right)$ between $98 \mathrm{~N}$ in loading stage and $98 \mathrm{~N}$ in unloading stage was analyzed to obtain HRC values. HRC values were calculated by $H R C=100-\left(\delta^{*} / 100\right)$.

MARC K6 was used as a FE code and MENTAT II as pre and post processors. True stress - logarithmic strain relationships were used in the FE calculation with large deformation, update and finite strain options [4]. Contact was judged when the indenter approached to the specimen in a distance less than $1.0 \times 10^{-5} \mathrm{~mm}$. The Coulomb friction below was employed to express a friction force in the analysis.

$$
F=\mu \cdot W
$$

where $F$ is the friction force, $\mu$ the friction coefficient and $W$ the normal force at a contact point. 


\section{Effect of inelastic material constants on HRC}

Effects of the friction coefficient and Young's modulus on HRC

Figure 2 shows an example of force - indenter displacement curve. The curve is approximated with a quadratic function as $P=a \delta^{2}+b \delta+c$, where $P$ is the load applied to the indenter and $\delta$ is the indenter displacement. In the Brinell analysis with a ball indenter, the curve was not quadratic but a linear relationship was found in the initial loading stage [2]. These results suggest that the type of indenter influences the shape of load - indenter curve.

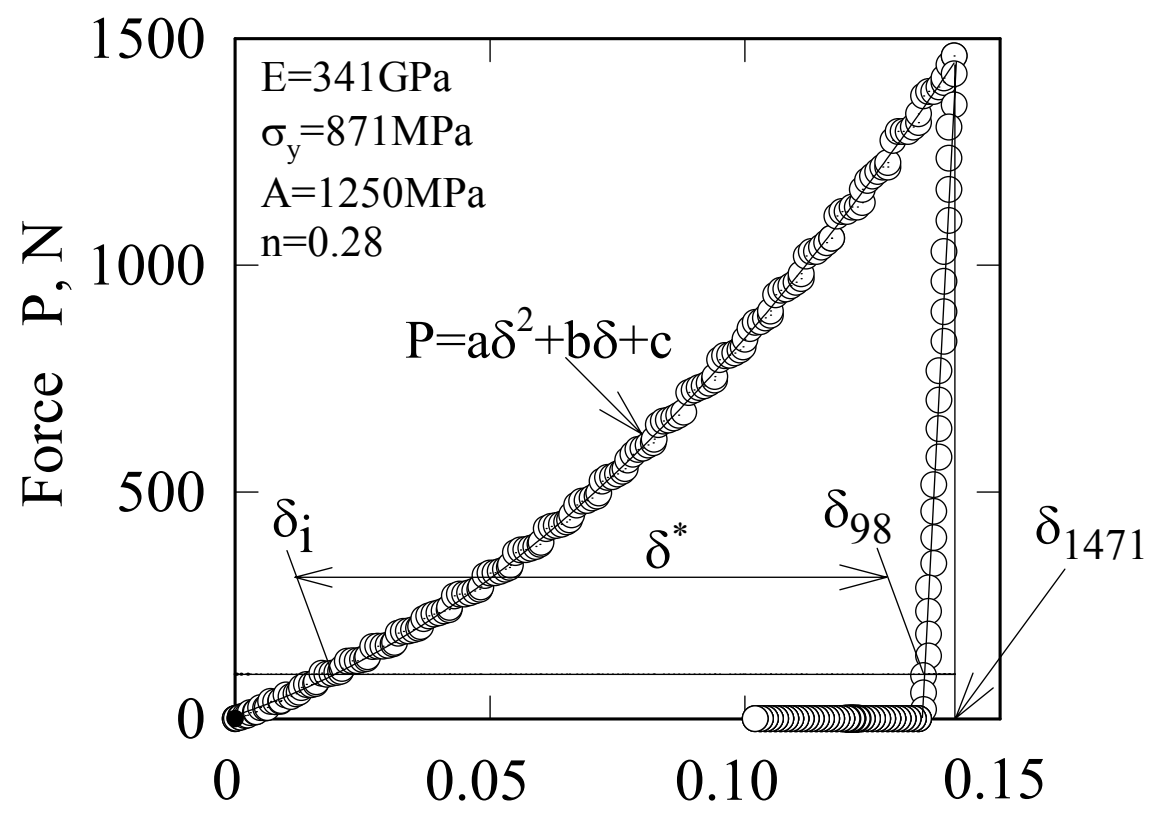

\section{Indenter displacement $\delta, \mathrm{mm}$}

Fig.2 Relationship between penetration force and indenter displacement.

This study assumes the power law inelastic constitutive equation expressed below

$$
\varepsilon_{p}=\left(\frac{\sigma-\sigma_{y}}{A}\right)^{\frac{1}{n}}
$$

where $\varepsilon_{p}, \sigma, \sigma_{\mathrm{y}}, \mathrm{A}$ and $\mathrm{n}$ are plastic strain, flow stress, yield stress and work hardening coefficient and exponent, respectively. These material constants of TAB6400 titanium alloy, INCONEL 718 nickel base superalloy and SCM 430 ferritic alloy are tabulated in Table 1 together with the hardness of those materials in FE analysis and experiments. The hardness in FE analysis is 7-8 \% smaller than that in experiments for TAB6400 and INCONEL 718 that have relatively large yield stresses. However, the difference is only $2 \%$ for SCM4320 where FE analysis gives a slightly smaller value than the experimental result. The satisfactory agreement shown in the table ensures the applicability of FE analysis to the simulation.

Table 1 Material constants and HRC in FE analysis and experiment.

\begin{tabular}{|c|c|c|c|c|c|c|c|}
\hline & $\mathrm{E}, \mathrm{GPa}$ & $\sigma_{\mathrm{y}}, \mathrm{MPa}$ & $\mathrm{A}, \mathrm{MPa}$ & $\mathrm{n}$ & $\begin{array}{c}\text { HRC } \\
\text { (Exp.) }\end{array}$ & $\begin{array}{c}\text { HRC } \\
\text { (FEM) }\end{array}$ & $\begin{array}{c}\text { HRC(Exp.) } \\
\text { HRC(FEM) }\end{array}$ \\
\hline TAB6400 & 105 & 870 & 694 & 0.411 & 33.3 & 31.0 & 1.07 \\
\hline INCONEL718 & 341 & 871 & 1250 & 0.280 & 44.2 & 41.0 & 1.08 \\
\hline SCM430 & 164 & 390 & 1053 & 0.217 & 25.6 & 25.0 & 1.02 \\
\hline
\end{tabular}




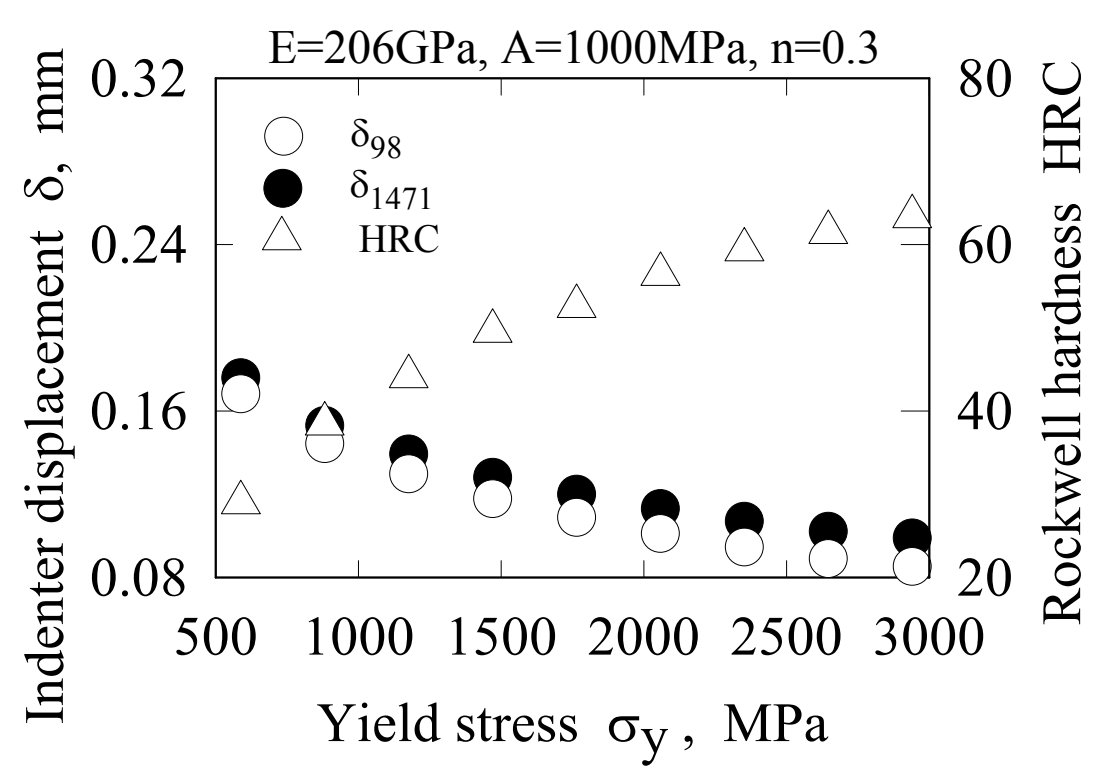

Fig.3 Variations of indenter displacement and hardness with yield stress.

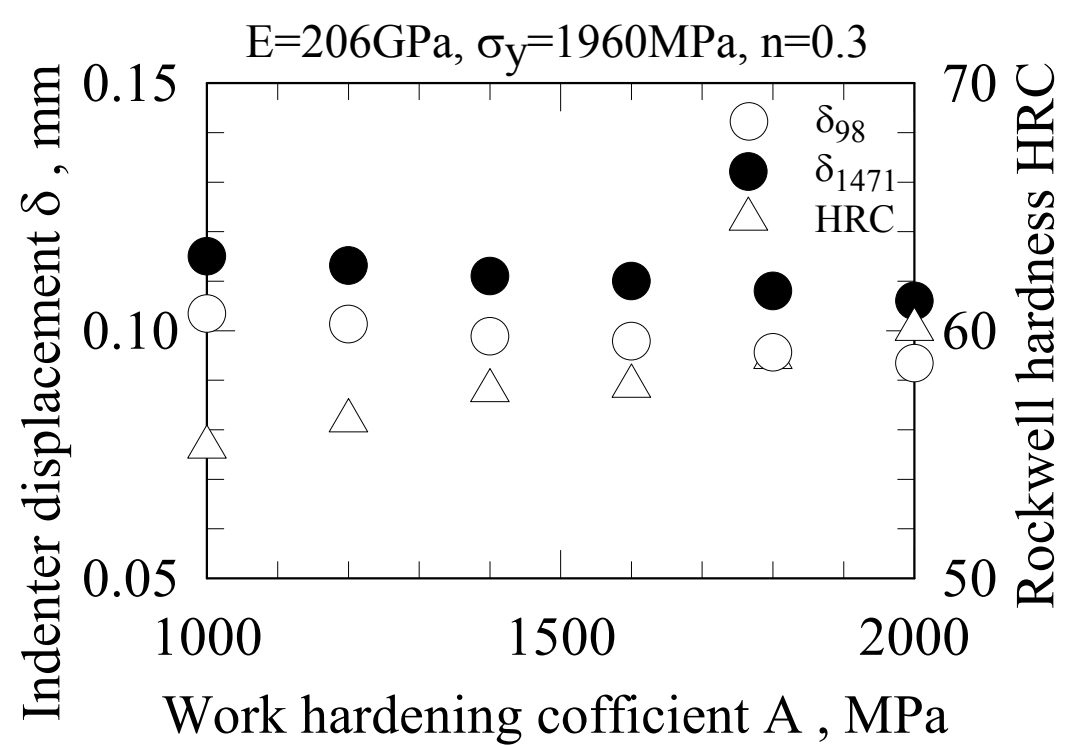

Fig.4 Variations of indenter displacement with strain hardening coefficient.

The variation of the hardness with yield stress is plotted in Figure 3 for $E=206 \mathrm{GPa}, \mathrm{A}=1000 \mathrm{MPa}$ and $\mathrm{n}=0.3$. The indenter displacements decrease with increasing yield stress and HRC increases accordingly. Yield stress has a significant effect on the hardness. Figure 4 depicts the effect of the strain hardening coefficient on $\mathrm{HRC}$ for $\mathrm{E}=206 \mathrm{GPa}, \sigma_{y}=1960 \mathrm{MPa}$ and $\mathrm{n}=0.3$. The indenter displacements linearly decrease with increasing $\mathrm{A}$ and $\mathrm{HRC}$ increases with increasing $\mathrm{A}$.

Figure 5 plots the effect of strain hardening exponent on the relative indenter displacement $\delta^{*}$ for respective $\sigma_{y}$ and $\mathrm{A}$ denoted in the figure. The relative displacement linearly increases with increasing the exponent for all the cases shown in the figure. The displacement takes smaller values at smaller $\sigma_{y}$ and smaller A. Since HRC inversely related with the relative displacement, HRC takes smaller values at smaller $\sigma_{y}$, smaller A and smaller n.

Quantitative formulation of HRC with inelastic material constants

As shown in Figure 5, the relative displacement $\delta^{*}$ can be equated as a linear logarithmic function of n, so the relationship is expressed as

$$
\delta^{*}=10^{\beta} n^{\alpha}
$$




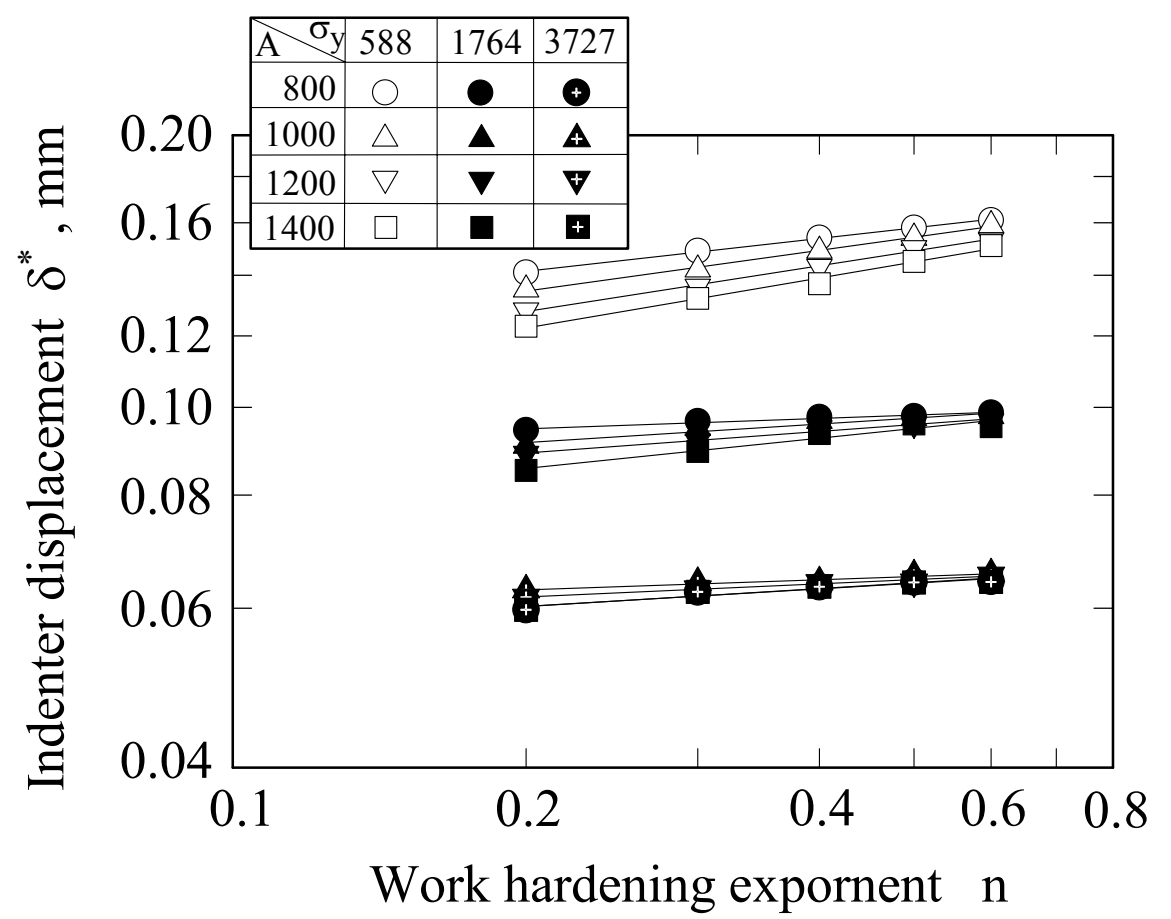

Fig.5 Variation of indenter displacement with strain hardening exponent.

$\alpha$ and $\beta$ are a function of $\sigma_{y}$ and $\mathrm{A}$.

The constants $\alpha$ and $\beta$ are equated as

$$
\begin{gathered}
\alpha=1.55 \times 10^{-8} \sigma_{y}^{2}-8.178 \times 10^{-5} \sigma_{y}+\left(1.09 \times 10^{-4} A+0.646\right) \\
A: \mathrm{MPa}, \quad \sigma_{:}: \mathrm{MPa}
\end{gathered}
$$

The exponent in equation(3) is only a function of and is expressed with a quadratic equation.

$$
\begin{gathered}
\beta=2.270 \times 10^{-8} \sigma_{y}^{2}-2.246 \times \sigma_{y}-0.6642 \\
\sigma_{:}: \mathrm{MPa}
\end{gathered}
$$

Substituting Eq. (4) and (5) into Eq. (3) gives the relationship between the relative displacement $\delta^{*}$ and the inelastic material constants as,

$$
\delta^{*}=10^{2.270 \sigma_{y}^{2}-2.246 \times 10 \sigma_{y}-0.6642} \cdot n^{1.155 \times 10^{-8}} \sigma_{y}^{2}-8.178 \times 10^{-5} \sigma_{y}+\left(1.09 \times 10^{-4} A+0.646\right)
$$

Figure 6 compares the predicted HRC by Eq.(6) with the FE results. In the figure, the hardness of the three materials is superimposed of which the predicted values are calculated using Eq. (6) from the inelastic material constants listed in Table 1. All the hardnesses including the experimental results are correlated within a narrow scatter band of a factor of 1.05. In the correlation in Figure 6, the inelastic material constants are ranged from 588 to $3727 \mathrm{MPa}$ for $\sigma_{y}$, from 800 to $1440 \mathrm{MPa}$ for A and 0.2 to 0.6 for $\mathrm{n}$. These ranges mostly cover the actually existing materials suitable for HRC testing. Thus, Eq.(6) is an appropriate equation for predicting HRC from the inelastic material constants for a wide range of matrials. Eq.(6) also indicates that how the inelastic material constants, yield stress and strain hardening coefficient and exponent, influence HRC value quantitatively.

\section{CONCLUSIONS}

1. Friction coefficient has no influence on Rockwell $\mathrm{C}$ hardness.

2. The maximum indenter displacement at $1471 \mathrm{~N}$ decreases with increasing Young's modulus. However, 


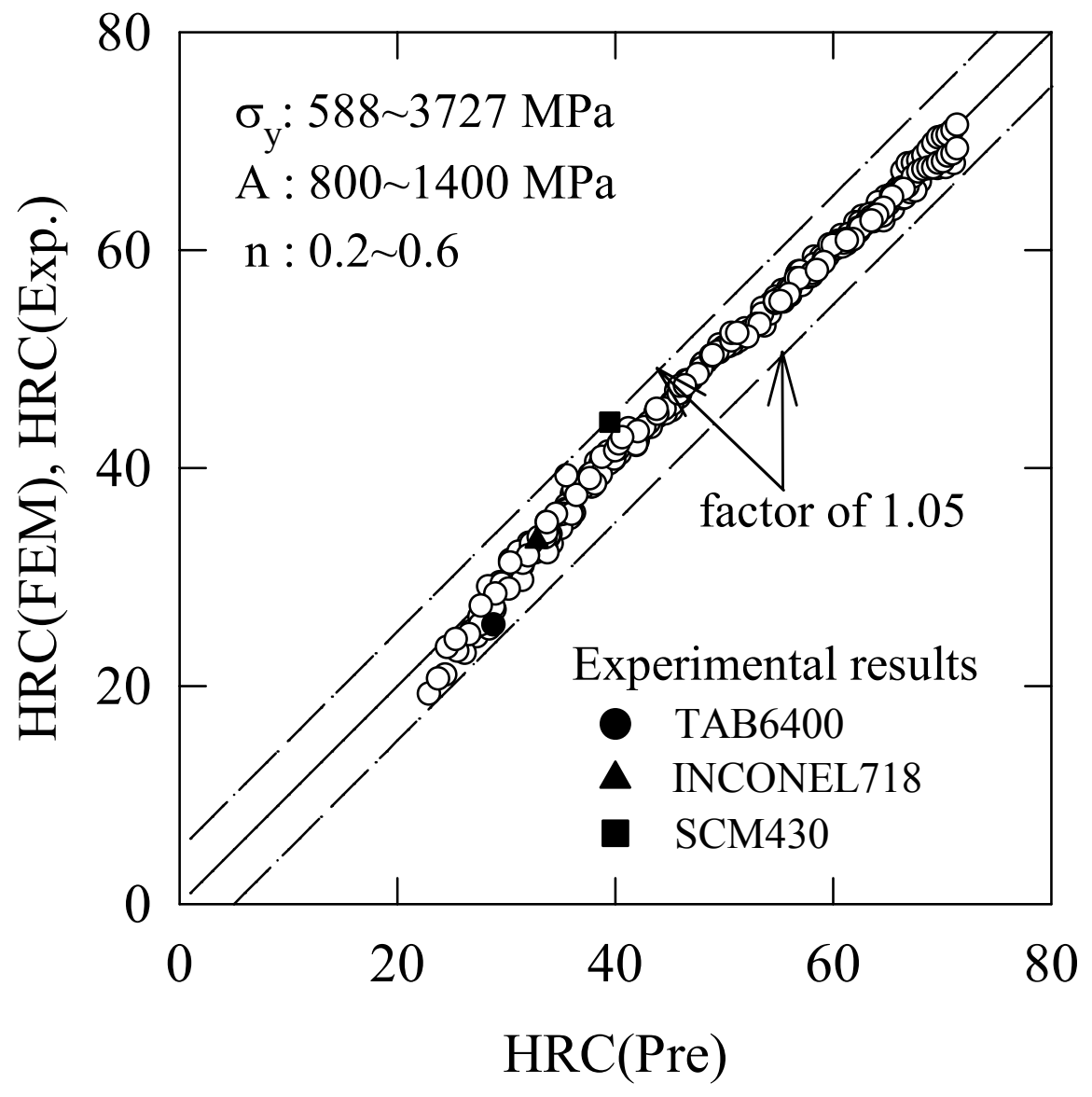

Fig.6 Comparison of HRC between FEM and experiment.

Young's modulus has no influence on Rockwell C hardness.

3. Rockwell $\mathrm{C}$ hardness increases with increasing yield stress and strain hardening coefficient. The hardness increases with decreasing strain hardening exponent.

4. Relative indenter displacement $\delta^{*}$ at $98 \mathrm{~N}$ between loading and unloading stages is equated with strain hardening exponent, $\mathrm{n}$, as $\delta^{*}=10^{\beta} n^{\alpha}$, where $\alpha$ and $\beta$ are functions of yield stress and strain hardening coefficient A expressed below.

$\alpha=1.55 \times 10^{-8} \sigma_{y}^{2}-8.178 \times 10^{-5} \sigma_{y}+\left(1.09 \times 10^{-4} A+0.646\right)$

$\beta=2.270 \times 10^{-8} \sigma_{y}^{2}-2.246 \times \sigma_{y}-0.6642$

The equations predicted the $\mathrm{FE}$ and the experimental results within a factor of 1.05 in the hardness range between 20 and 70 .

\section{References}

1.Yoshizawa, T.(1967), “Hardness Testing and Its Applications”, Shokado Pub., Tokyo.

2. Japan Industrial Standards, JIS Z 2245 (1997) , "Rockwell Hardness Test ".

3.Hamada, N., and Sakane, M.(1999), Trans. Japan Society of Mechanical Eng., A63, 254.

4.Marc (1995), “Mark User Information K-6", C3-177. 